\title{
Scaling of the Hysteresis Loop in Two-dimensional Solidification
}

\author{
K. Budde ${ }^{a}$, I. Lyuksyutov ${ }^{c *}$, H. Pfnür ${ }^{a * *}$, G. Godzik ${ }^{a}$ and H.-U. Everts ${ }^{b}$ \\ ${ }^{a}$ Institut für Festkörperphysik and ${ }^{b}$ Institut für Theoretische Physik, Universität Hannover, \\ Appelstraße 2, D-30167 Hannover, Germany \\ ${ }^{c}$ Department of Physics, Texas A\&M University, \\ College Station, TX 77843-4242, USA
}

(July 1, 2018)

\begin{abstract}
The first order phase transitions between a two-dimensional $(2 \mathrm{~d})$ gas and the $2 \mathrm{~d}$ solid of the first monolayer have been studied for the noble gases $\mathrm{Ar}, \mathrm{Kr}$ and $\mathrm{Xe}$ on a $\mathrm{NaCl}(100)$ surface in quasiequilibrium with the three-dimensional (3d) gas phase. Using linear temperature ramps, we show that the widths of the hysteresis loops of these transitions as a function of the heating rate, $r$, scales with a power law $\propto r^{\alpha}$ with $\alpha$ between 0.4 and 0.5 depending on the system. The hysteresis loops for different heating rates are similar. The island area of the condensed layer was found to grow initially with a $t^{4}$ time dependence. These results are in agreement with theory, which predicts $\alpha=0.5$ and hysteresis loop similarity.

68.10Jy, 68.35.Rh, 61.14.Hg
\end{abstract}

Adsorption isotherm measurements are long known to give detailed information about adsorbated-substrate interactions as well as about lateral interactions, if combined with structural information. They were first introduced into surface studies by Langmuir 11]. In most cases, adsorption is a first order phase transition [2] with the heat of adsorption as the latent heat, which is expected to show the typical hysteresis behavior due to overheating and undercooling the adsorbed layer. In this Letter we want to concentrate on a specific aspect of hysteresis, scaling of the energy turn-over as a function of frequency and amplitude, as measured by the hysteresis loop area.

Scaling of the hysteresis loop area by cycling through a first order phase transition has been predicted long ago and has been studied in detail in ferromagnetic systems. Already in early pioneering work on a 3D magnet, which dates back to the last century [3], first indications of scaling behavior of the hysteresis loop area have been found. Recently, experimental investigations of hysteresis loop scaling in ultrathin magnetic films have been performed [4], [5], [6]. However, although power law behavior is found in all studies [- 4 , [5], [6], the effective exponents obtained differ by typically one order of magnitude between theoretical models [7] and measurements of energy loss scaling in ultrathin magnetic films (see discussion in Ref. [6]). No obvious explanation for this large discrepancy has come up yet.

Universality mean that scaling of the hysteresis loop area to be observable not only in ferromagnetic systems, but also, e.g., in adsorbed layers, for which, according to our knowledge, this property has not been studied in any detail yet. This class of systems is characterized not only by completely different parameters, but also by narrow domain walls of just a few lattice constants, in contrast to magnetic systems. As a result nucleation barriers in adsorbed layers can be much smaller than in magnetic films. We have recently predicted scaling behavior of the adsorption hysteresis loop width, $\mu_{h}$, and of the hys- teresis loop area $\mathcal{A}[\mathbb{8}$ as a function of amplitude and cycle frequency for the situation close to thermodynamic equilibrium. A more detailed analysis of the hysteresis behavior for the case of magnetic film [9], [10] predicts also similarity of different hysteresis loops.

For our test experiments of scaling of hysteresis loops in adsorption of two-dimensional layers, we used the phase transition to condensation of a $2 \mathrm{~d}$ solid of the noble gases $\mathrm{Xe}, \mathrm{Kr}$ and $\mathrm{Ar}$ on single crystalline thin films of $\mathrm{NaCl}(100)$ in quasi-equilibrium with the $3 \mathrm{~d}$ gas phase at room temperature. Due to the van-der-Waals attraction between the noble gas atoms, this transition is strongly first order, and can be easily measured, as shown below. The deviation of the chemical potential from the equilibrium value at the phase transition, $\mu$, in the case of adsorption plays the role of the magnetic field, which was periodically varied around the equilibrium value with amplitude $\mu_{0}$.

In our model, the process of adsorption is divided into two stages: nucleation of islands and growth of these islands. The situation when the nucleation time $\tau_{n} \ll \tau$, the cycle time, has been considered in Ref. [8]. We call this situation growth controlled hysteresis. For heterogeneous nucleation this condition can be easily fulfilled.

With the additional assumption that an island with area $\mathcal{A} \propto L^{2}$ grows with a boundary velocity $v(\mu)$ that depends on $\mu$ like $v=\Gamma \mu$, we obtain for the island of diameter $L$

$$
L(t)-L_{0}=2 C \tau \Gamma \mu_{0}\left(\frac{\mu(t)}{4 \mu_{0}}\right)^{2}=\frac{C \Gamma}{2} r t^{2},
$$

where $L_{0}$ is of the order of the size of a stable nucleation center, $\mu(t)=r t$ with linear heating rate $r$ (adapted according to the experiments carried out), and $\mathrm{C}$ a constant of order unity. We have explicitly used that $\left(\tau_{n} / \tau\right)^{2} \ll 1$. Eq.1 1 shows that the system behavior is controlled by the characteristic length $L_{\tau}=\tau \Gamma \mu_{0}=4 \mu_{0}^{2} \Gamma / r$. With increase of $L_{\tau}$ the area of the adsorption-desorption cycle 
loop will decrease. If $L_{N}$ is the mean distance between nucleation centers, then the hysteresis parameters are defined by the ratio $L_{N} / L_{\tau}$ [9], [8].

From Eq.1 the scaling behavior of the hysteresis loop width $\mu_{h}$ and of the area of the adsorption-desorption cycle loop $\mathcal{A}$ as functions of $\tau$ and $\mu_{0}$ can be evaluated in the limit $L_{N} \ll L_{\tau}$, i.e. when $L_{N}$ determines the maximum island size. At $\mu=\mu_{h} / 2$ the typical island size $L \approx L_{N}$. The condition $L_{N} \ll L_{\tau}$ requires that $\mu_{h} \ll \mu_{0}$ and $L_{N} \approx\left(\Gamma \tau / \mu_{0}\right)\left(\mu_{h}\right)^{2}$. If we assume that $L_{N}$ does not depend significantly on $\tau$ and $\mu_{0}$, we immediately obtain that $\mu_{h}$ scales as $\mu_{h} \propto \sqrt{\mu_{0} / \tau} \propto \sqrt{r}$. Under the same conditions of $L_{N}$ independent (or weakly dependent) on $\mu_{0}$ and $\tau$, the hysteresis loops are similar and scale in the same way as $\mu_{h}$. This has been predicted in [9]. At sufficiently low frequencies, the height of hysteresis loop does not depend on frequency.

This part of the frequency dependence is tested in our experiments, in which we linearly ramp the surface temperature up and down, keeping the ambient gas pressure constant. In addition to scaling of the hysteresis loop area, the time dependence of island sizes can be tested by experiment, which, according to eq. 1 1 should grow as $L(t) \propto r t^{2}$. As long as the islands don't touch each other, the coverage $\Theta$ depends on $L$ as $\Theta \propto L^{2} \propto r^{2} t^{4}$. In a diffraction experiment, the integrated intensity of a superstructure spot should be proportional to the coverage so that this equation can be tested easily measuring the initial time dependence of the integrated diffracted intensities.

Our experiments were carried out in a UHV-chamber equipped with high resolution LEED supplemented by a high resolution optical detector [11], Auger spectrometer and a quadrupole mass spectrometer, at a base pressure of $2 \times 10^{-11}$ mbar. Peak intensities of LEED diffraction spots were both measured with a Faraday cup and with the optical detector, while integrated intensities were obtained from images taken from the optical detector using a high resolution slow scan CCD camera. The $\mathrm{NaCl}(100)$ surfaces were prepared in situ as 3 double layers thick epitaxial films by evaporation of $\mathrm{NaCl}$ onto a $\mathrm{Ge}(100)$ substrate at a surface temperature of $200 \mathrm{~K}$ and subsequent annealing to $550 \mathrm{~K}$. This procedure produces single crystalline $\mathrm{NaCl}$ layers that are in registry with the Ge substrate on the flat terraces and overgrow monoatomic steps of the Ge substrate in a carpet-like mode [12]. Therefore the quality of the films is determined by the step density of the substrate, which had typical terrace length of $400 \AA$. The Ge substrates (size $10 \times 10 \times 1 \mathrm{~mm}$ ) were oriented with a precision of $0.1^{\circ}$ using a Laue camera and polished with diamond pastes and a final chemomechanical treatment. These samples were mounted onto a He-cooled flow-cryostat. They were heated either by electron bombardment or radiation. For the measurements only radiation was used. Temperature was measured using a Chromel-Alumel thermocouple attached to

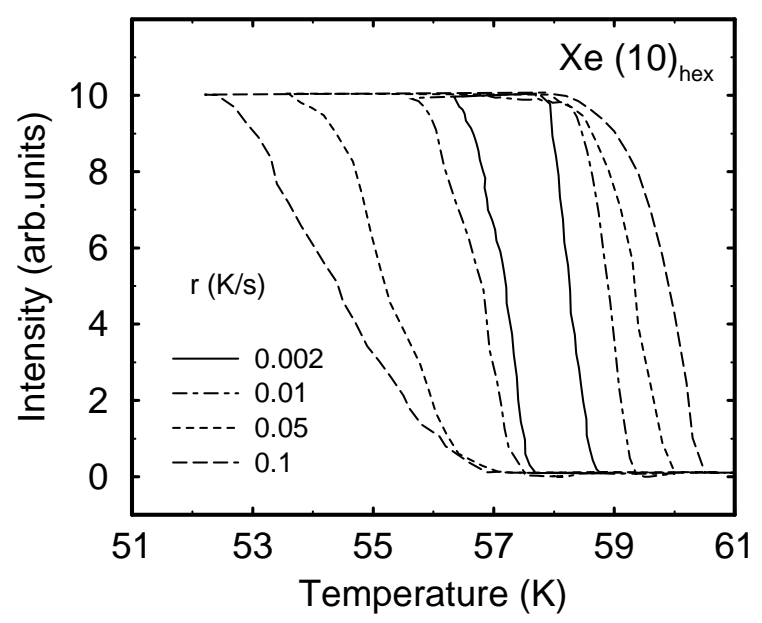

FIG. 1. Hysteresis loops of the 2d gas-solid phase transition of $\mathrm{Xe} / \mathrm{NaCl}(100)$ at a Xe pressure of $10^{-7}$ mbar. The peak intensity of the of a first order superstructure spot was monitored at the heating rates indicated.

the base plate of the mounting assembly, which was in good thermal contact with the Ge substrate. A computerized temperature controller with a nominal resolution of $0.001 \mathrm{~K}$ was used for linear temperature ramps up and down. High purity gases were dosed directly onto the sample through a ring-shaped slit centered around the surface normal, which could be cooled to $100 \mathrm{~K}$ with $\uparrow \mathrm{N}_{2}$. Measurements carried out both with gas at room temperature and at $100 \mathrm{~K}$ showed that the gas temperature had a negligible influence on the results presented below. Pressures given below are pressures obtained from a calibration with gas exposures from background gas. Homogeneity of the pressure on the surface was also tested and found to vary negligibly over the surface area seen by the LEED beam.

$\mathrm{Xe}$ on $\mathrm{NaCl}(100)$ condensed in the first monolayer forms a quasi-hexagonal incommensurate structure 13 so that superstructure diffraction spots were directly used to measure hysteresis both with integrated and peak intensities. While the first monolayer of Ar forms an ordered $(1 \times 1)$ structure, a diffuse $(2 \times 1)$ structure with glide plane symmetry is seen for $\mathrm{Kr}$. Only peak intensities of integral order beams have been evaluated for the latter two systems. Data of equivalent beams have been averaged where available.

Typical data of the hysteresis during condensation of the quasi-hexagonal Xe layer are shown in Fig. 1. The linear heating rate was varied by two orders of magnitude between 0.001 and $0.1 \mathrm{~K} / \mathrm{s}$. This rate was limited at small rates by the resolution of temperature measurement, at high rates by the onset of intermixing of second layer condensation. Measurements were taken for gas pressures of $10^{-6}$ and $10^{-7}$ mbar. Not surprisingly, slopes during ad- 


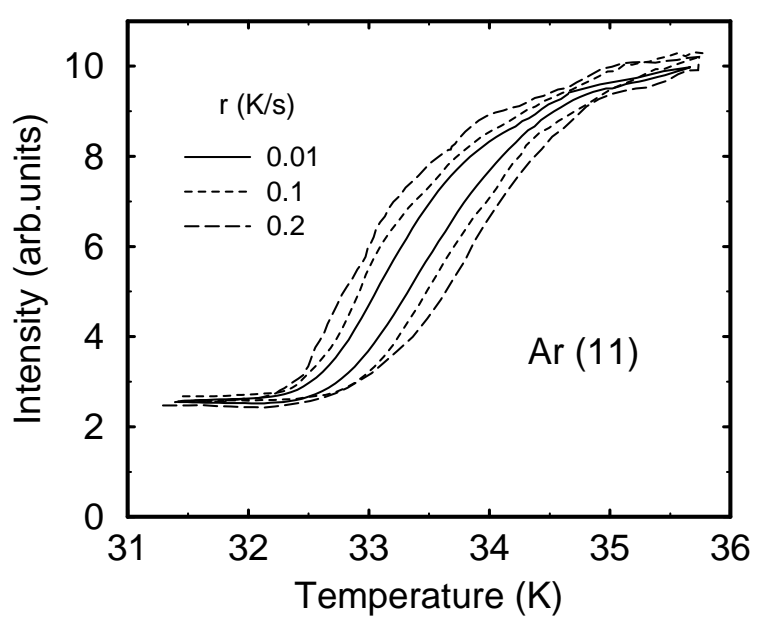

FIG. 2. Hysteresis loops of the $2 \mathrm{~d}$ gas-solid phase transition of $\mathrm{Ar} / \mathrm{NaCl}(100)$. The change of peak intensity of an first order integral spot was monitored for the $2 \mathrm{~d}-(1 \times 1)$ Ar solid formed.

sorption and desorption are not symmetrical due to the exponential dependence of the desorption rate on surface temperature. At the highest rate the form during condensation changes due to the mentioned onset of intermixing with second layer adsorption, leading also to a decrease of intensity with decreasing temperature. Inside these limits of heating rate the intensity at saturation did not depend on the heating rate.

Similar measurements were taken measuring the condensation induced changes of peak intensities of integral order beams for $\mathrm{Ar}$ and $\mathrm{Kr}$ on $\mathrm{NaCl}$, which, depending on electron energy of the LEED experiment and on diffraction order, can be both positive or negative. An example for Ar induced intensity changes is shown in Fig. 2. Please note that the dependence on noble gas concentration need not be linear in these cases.

For all three systems, we evaluated the dependence of the widths of the hysteresis loops at half maximum intensity on the heating rate $r$. The results are shown as a log-log plot in Fig. 3. Power laws were obtained for all three systems. The effective exponents $\alpha$ obtained from these plots are close to 0.4 for Xe and $\mathrm{Ar}$ condensation (within the statistical uncertainty of about 10\%), whereas the average value for $\mathrm{Kr}$ of $\alpha=0.50$ actually corresponds exactly to the value expected from the simple model of growth controlled hysteresis already mentioned. Also the experimental results of Xe and Ar are sufficently close to this value so that this model seems to describe the essential physics correctly.

A further test of this model can be carried out by analyzing the time dependence of island growth in the initial stages of growth. For this purpose we plotted the integrated intensities of the Xe systems as a function of time during condensation again on log-log scale (see Fig 1). As

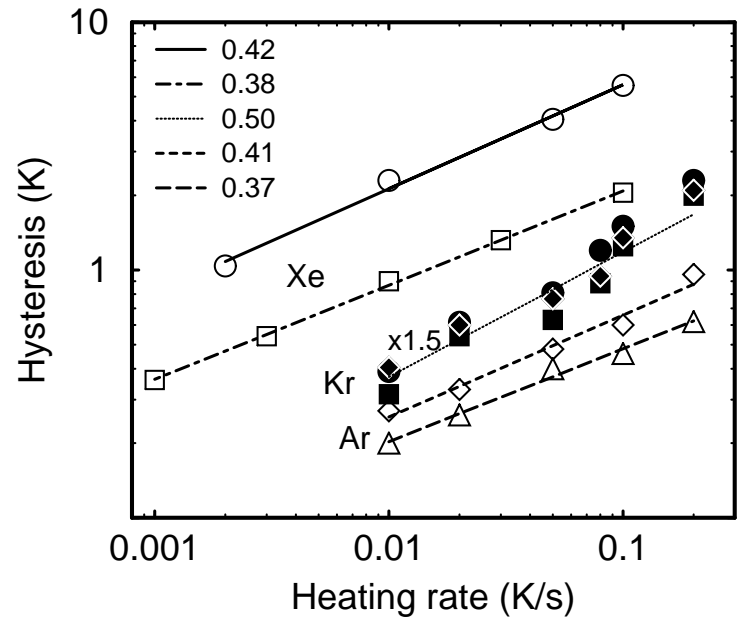

FIG. 3. Log-log plots of the width of hysteresis loops, $\Delta T$, as a function of the heating rate $r$ in presence of a $3 \mathrm{~d}$ gas pressure. $\mathrm{Xe} / \mathrm{NaCl}(100): \quad p=1 \times 10^{-7} \mathrm{mbar}(\bigcirc)$, $p=1 \times 10^{-6}$ mbar ( $\left.\square\right)$. Full symbols: $\mathrm{Kr} / \mathrm{NaCl}(100)$ at $p=1 \times 10^{-7} \mathrm{mbar}$ (three different orders of diffraction). $\diamond$ and $\triangle: \operatorname{Ar} / \operatorname{Nacl}(100)$ at $p=1 \times 10^{-7}$ mbar measured for the (10) and (11) beams, respectively.

mentioned, the model predicts the integrated intensity to increase $\propto r^{2} t^{4}$, i.e. $\propto(\Delta T)^{4} / r^{2}$. Therefore, the data in this figure are plotted once as a function of $\Delta T$, and a second time scaled by $r^{2}$. The integrated intensities follow closely the $t^{4}$ dependence predicted by our simple model, but deviates from it when coverage gets closer to saturation. Of course, this plot is very sensitive to the choice of time zero, for which we chose the condensation temperature at equilibrium, as estimated from the center of the hysteresis curves. This is the earliest possible time. It is fully consistent with the assumptions of the model used, which assumes a small nucleation time $\tau_{n}$. The intensity zero was taken as the bottom of the hysteresis curves without further adjustments. This result therefore seems to nicely corroborate the model assumption of growth controlled hysteresis, which is not limited by diffusion on the surface.

These results turned out to be insensitive to additional production of anion vacancies on the $\mathrm{NACl}$ substrate, which is caused by the measuring electron beam. They act as additional nucleation centers. Though they reduce the maximum size of islands, they obviously do not change the growth modes, in agreement with the expectations from our model. For larger islands, diffusion to the island boundaries of course can no longer be neglected resulting in deviations from our model. Diffusion is particularly important in the second layer on already condensed islands, since there the sticking coefficient is much larger than on the bare surface [12]. This process increases the speed of growth, especially at the initial stages. Although in a real experiment mass transport to 


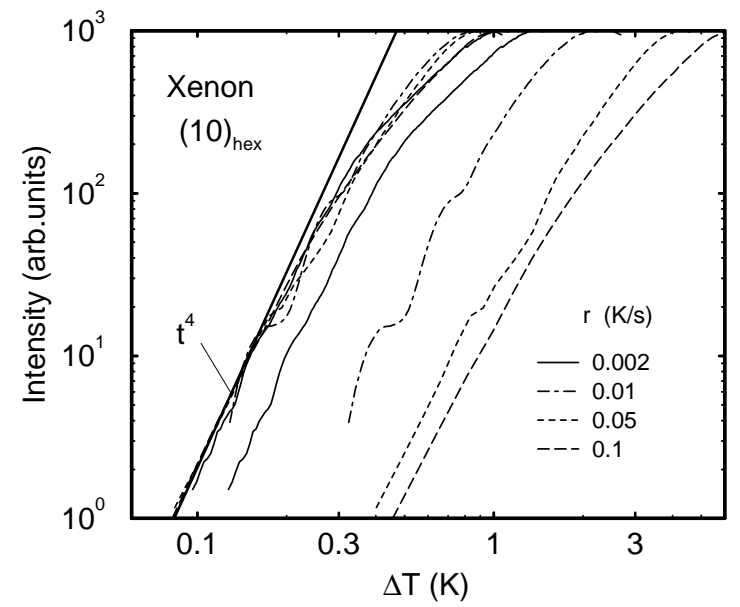

FIG. 4. Test of scaling with time of integral intensities of a first order superstructure spot of $\mathrm{Xe} / \mathrm{NaCl}(100)$, The curves close to the straight line have been divided by $r^{2}$.

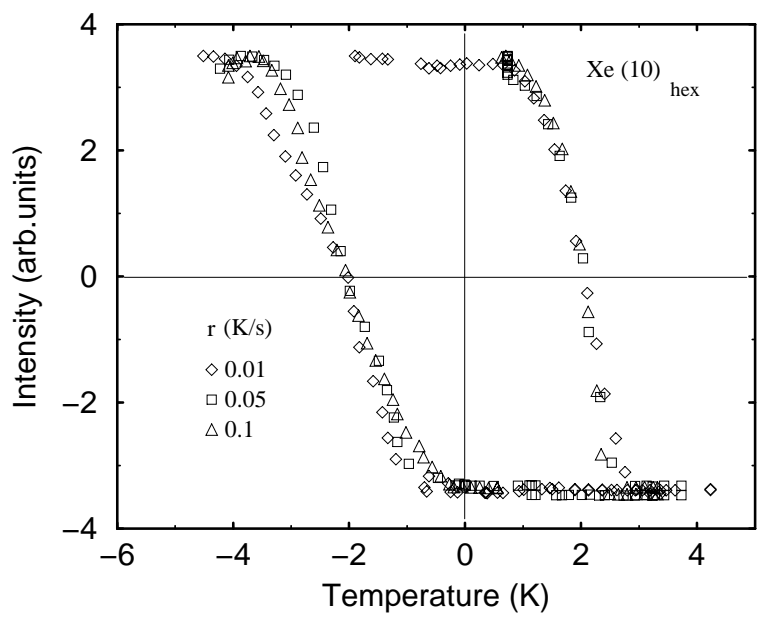

FIG. 5. Hysteresis curves for Xe rescaled by $(\Delta T)^{-\alpha}$.

the growing boundary by diffusion is always present, we therefore expect that our main result -dynamical scalingremains valid for conditions close to equilibrium.

An explicit test of similarity of the hysteresis curves was again carried out for $\mathrm{Xe} / \mathrm{NaCl}(100)$. The rescaled curves (after centering) are shown in Fig. 5 for one order of magnitude changes in the heating rate. While the evaporation data fit perfectly to a common line, there is more scatter in the data during condensation but no general trend for the small deviations was found. Therefore, also similarity seems to be fulfilled by these data.

To conclude, we have introduced a new method of studing adsorption phenomena, observed scaling behavior for adsorption, found corresponding critical exponents and proved similarity of hysteresis loops. Our studies of scaling of the hysteresis loop areas in adsorption gave re- sults in almost quantitative agreement with theoretical expectations using a simple model. This situation is in sharp contrast with the situation in magnetic films. Possible reasons are the small width of the interface between islands (of the order of one lattice constant) and/or a low interface energy. The latter would cause small nucleation barriers. It is consistent with the dominant mechanism of growth controlled hysteresis found in our experiments. For studies of hysteresis phenomena, we have shown that adsorbed films are complementary to ultrathin magnetic films, since they open a completely different parameter space. The method we have used can be extended to a broad range of 2-D gas - 2-D solid phase transitions. In addition this type of measurements can give important information about dynamics of first order phase transitions on surfaces and about 2-D interface motion.

We wish to thank V.L.Pokrovsky with whom we extensively discussed the problem of hysteresis loop scaling. The work was supported by the Niedersächsische Ministerium für Wissenschaft and Kultur, by Volkswagen Stiftung and by the Deutsche Forschungsgemeinschaft. One of us (I.L.) was partly supported by a DOE grant (DE-FG03-96ER 45598).

* $\quad$ Also at Institute of Physics, 252028 Kiev, Ukraine.

** corresponding author; e-mail: pfnuer@fkp.uni-hannover.de

[1] A. Zangwill, Physics at Surfaces, Cambridge University Press, Cambridge 1988.

[2] L. W. Bruch, M. W. Cole and E. Zaremba, Physical Adsorption: Forces and Phenomena, Clarendon Press, Oxford 1997.

[3] C.P.Steinmetz, Trans.Am.Inst.Electr.Eng. 9, 3 (1892).

[4] Y.-L. He and G.-C. Wang, Phys. Rev. Lett. 70, 2336 (1993).

[5] Q. Jiang, H.-N. Yang, and G.-C. Wang, Phys. Rev. B 52, 14911 (1995).

[6] J.-S. Suen and J. L. Erskine, Phys. Rev. Lett. 78, 3567 (1997).

[7] References to theoretical work can be found in [6] and 10.

[8] I. F. Lyuksyutov, H. Pfnür and H.-U. Everts, Europhys. Lett. 41, 395 (1998).

[9] I. F. Lyuksyutov, T. Nattermann and V. L. Pokrovsky cond-mat/9709194

[10] I. F. Lyuksyutov, T. Nattermann and V. L. Pokrovsky cond-mat/9802065

[11] K. Budde and H. Pfnür, (unpublished).

[12] C. Schwennicke, J. Schimmelpfennig and H. Pfnür, Surf. Sci. 293, 57 (1993).

[13] C. Schwennicke, J. Schimmelpfennig and H. Pfnür, Phys. Rev. B 48, 8928 (1993). 